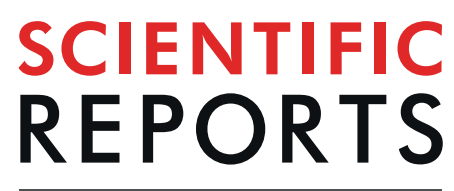

natureresearch

\title{
Compensating Atmospheric Channel Dispersion for Terahertz Wireless Communication
}

\author{
Karl Strecker, Sabit Ekin (1) \& John F. O’Hara (1)* \\ We report and demonstrate for the first time a method to compensate atmospheric group velocity \\ dispersion of terahertz pulses. In ultra-wideband or impulse radio terahertz wireless communication, \\ the atmosphere reshapes terahertz pulses via group velocity dispersion, a result of the frequency- \\ dependent refractivity of air. Without correction, this can significantly degrade the achievable data \\ transmission rate. We present a method for compensating the atmospheric dispersion of terahertz \\ pulses using a cohort of stratified media reflectors. Using this method, we compensated group velocity \\ dispersion in the 0.2-0.3 THz channel under common atmospheric conditions. Based on analytic \\ and numerical simulations, the method can exhibit an in-band power efficiency of greater than \\ $98 \%$ and dispersion compensation up to $99 \%$ of ideal. Simulations were validated by experimental \\ measurements.
}

The capacity of wireless data links has risen exponentially in the last decade ${ }^{1-3}$, yet demand for higher data rates continues to increase. In the coming years, this increase is expected to accelerate ${ }^{4,5}$, even beyond the capacity of fifth-generation $(5 \mathrm{G})$ wireless $^{6-8}$. It has become apparent that the next generation of wireless communication technologies must push operating frequencies into the terahertz $(\mathrm{THz})$ range to satisfy this predicted demand ${ }^{3,8,9}$. The development of any technology improving the bit rate-distance product or signal-to-noise ratio (SNR) of THz wireless links is an important step forward.

For THz wireless communications, there are two fundamental factors that limit achievable data rate and SNR. The most familiar is absorption, which limits SNR and is caused predominantly by rotational resonances of water vapor in the atmosphere ${ }^{10-13}$. These resonances also directly impact data rate by introducing group velocity dispersion (GVD). GVD arises when the many frequency components in a broadband signal propagate at different velocities due to the frequency-dependent refractive index of the channel medium. In the THz regime (0.1-10 $\mathrm{THz}$ ), the atmosphere itself is one such dispersive channel ${ }^{14,15}$. Because of GVD, single data bits, notionally represented by transform-limited $\mathrm{THz}$ pulses, spread temporally out of their assigned bit slot, superposing with bits in neighboring slots, resulting in inter-symbol interference (ISI). One solution to mitigate ISI is dilation of the bit slot, but this decreases the overall data rate ${ }^{16}$ and is clearly undesirable.

GVD is an emerging problem in THz wireless systems. Unlike in microwave channels, water vapor resonances in the $\mathrm{THz}$ regime are very strong, and the available bandwidths are massive $\left(60-180 \mathrm{GHz}^{15}\right)$. Accordingly, it has been shown that GVD has a significant impact on $\mathrm{THz}$ wireless data rates ${ }^{15}$. From previous work and our own calculations, reductions in data rate by a factor of 3 times or more are possible in single links over realistic signal ranges (1-20 km $\left.{ }^{17-19}\right)$. Specifically, GVD will become limiting whenever the combination of link distance, atmospheric water vapor density, and channel bandwidth exceeds a certain minimum threshold, still to be defined. The majority of experimental THz links reported to date have not reached this threshold, either being long-range and low bandwidth ${ }^{17-19}$, or short-range and high bandwidth ${ }^{20,21}$. However, there is no fundamental limitation that prevents future technologies from exceeding this limit. When they do, GVD management will become a critical factor in overall channel performance, just as it was in earlier generations of fiber-optic systems ${ }^{16}$.

One particular application where dispersion control may rapidly become critical is in the point-to-point distribution of ultra-high definition (UHD) and 3-D video ${ }^{22}$. For UHD video, uncompressed (real-time) data rates of up to $25 \mathrm{Gbps}$ (giga bits per second) are required. For 3-D video, the data rate can reach $100 \mathrm{Gbps}^{20}$. In a configuration analogous to fiber-optic systems with doped-fiber amplifiers ${ }^{16}$, a series of point-to-point, wireless $\mathrm{THz}$ links might carry this high-bandwidth signal over tens of kilometers using repeater stations (which are generally more economical than full receivers). For such systems, dispersion would accumulate over the full distance of the 


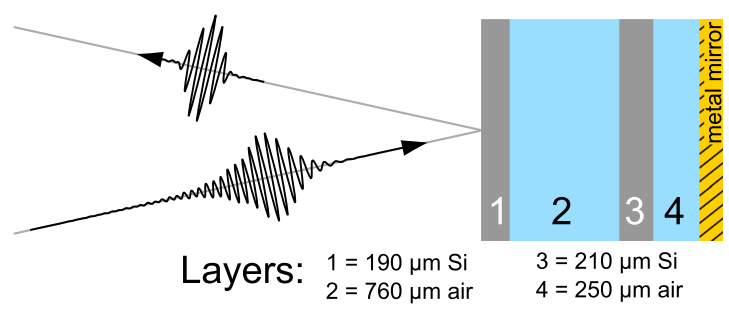

Figure 1. Silicon-based MGTI design and concept. Temporally dispersed terahertz waves reflect from the MGTI and experience dispersion opposite that of the atmospheric channel, thus restoring their short pulsewidth.

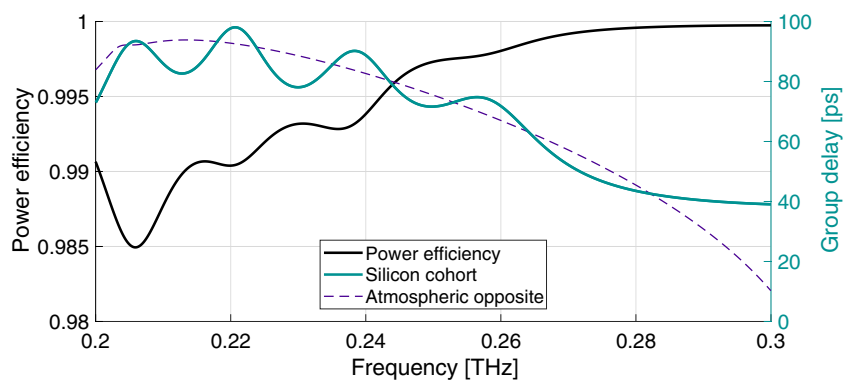

Figure 2. Analytic performance of Si-MGTI cohort. The dashed line indicates the target group delay, the opposite of atmospheric group delay at $\rho_{\mathrm{wv}}=10.37 \mathrm{~g} / \mathrm{m}^{3}$ for a $4 \mathrm{~km}$ propagation range. The thick black curve indicates the overall power efficiency. Plots show overall effects of one reflection from each MGTI of the cohort.

link, easily causing a reduction in data rate by an order of magnitude or more. In this case, dispersion management becomes critical.

In this report, we present a method and device for compensating GVD in THz wireless communication channels that is highly effective, offers zero latency, consumes no power, and has low insertion loss. Using our approach in the $0.2-0.3 \mathrm{THz}$ frequency channel, we demonstrate a compensation of up to $99 \%$ of the GVD experienced by a transform-limited $\mathrm{THz}$ pulse propagating through $4 \mathrm{~km}$ of atmosphere with a water vapor density of $\rho_{\mathrm{wv}}=10.37 \mathrm{~g} / \mathrm{m}^{3}\left(60 \%\right.$ relative humidity at $\left.20^{\circ} \mathrm{C}\right)$. Moreover, our approach can be implemented in a monolithic device that can simply be inserted into the THz beam to correct the signal GVD, and that has an insertion loss as low as $0.07 \mathrm{~dB}$ (over $98 \%$ power efficient). To our knowledge, this is the first attempt to correct atmospheric GVD in the $\mathrm{THz}$ regime.

\section{Results}

To compensate atmospheric dispersion, a device must be introduced into the THz beam that has GVD equal but opposite to that of the atmosphere. The resulting overall channel, comprised of both the atmosphere and the device, thus exhibits a cumulative GVD approaching zero. Our method uses a minimal structure of stratified dielectrics, arranged strategically in front of a high-reflector to form a multiple Gires-Tournois interferometer (MGTI) at THz frequencies. The dielectrics alternate between low and high refractive index, and the resonant trapping of frequencies within the structure introduces a frequency-dependent group delay. The optical thickness of each layer is tuned such that the overall GVD of the device matches the atmospheric opposite over a broad bandwidth. The THz wave experiences this opposite GVD during reflection from the MGTI.

The general MGTI structure is illustrated in Fig. 1. The parallel MGTI dielectric multilayers are readily modeled by means of characteristic matrices ${ }^{23}$ and the overall structures may be designed and rapidly optimized using genetic algorithms. For purposes of design and simulation, it is convenient to use group delay instead of GVD, since the former is specific to a set of channel conditions, whereas GVD is normalized to channel length. The curve labeled "Atmospheric Opposite" in Fig. 2 shows the ideal group delay profile for compensating a $4 \mathrm{~km}$ path length through an atmosphere with uniform water vapor density $\rho_{\mathrm{wy}}=10.37 \mathrm{~g} / \mathrm{m}^{3}\left(60 \%\right.$ relative humidity at $20^{\circ}$ C) over the entire $100 \mathrm{GHz}$ band centered at $0.25 \mathrm{THz}$. As detailed in the methods section, the absorption and refractivity of the atmosphere were calculated using Molecular Response Theory ${ }^{11,12,24}$ and water vapor resonance line data from the HITRAN database ${ }^{25}$.

While Fig. 1 shows only one design, two different MGTIs were actually employed together to compensate the above described dispersion. Using a cohort of two MGTIs increases the effectiveness of compensation by improving the fit to the atmospheric opposite over a larger bandwidth. Each MGTI was designed with alternating layers of high-resistivity silicon $(\mathrm{Si})$ and air. The layer thicknesses were obtained by using a genetic algorithm to compute the optimal characteristic matrix for a cohort of two MGTIs in reflection. The optimal layering for the first MGTI was $190 \mu \mathrm{m}$ of silicon, $760 \mu \mathrm{m}$ of air, $210 \mu \mathrm{m}$ of silicon, $250 \mu \mathrm{m}$ of air, and finally the reflector. Thickness variations of $\pm 5 \mu \mathrm{m}$ had minor effects to overall performance. The silicon was assumed to be lossless with a refractive index of $n_{\mathrm{Si}}=3.418$. For the second MGTI, the layering was $210 \mu \mathrm{m}$ of silicon, $800 \mu \mathrm{m}$ of air, $260 \mu \mathrm{m}$ of silicon, 


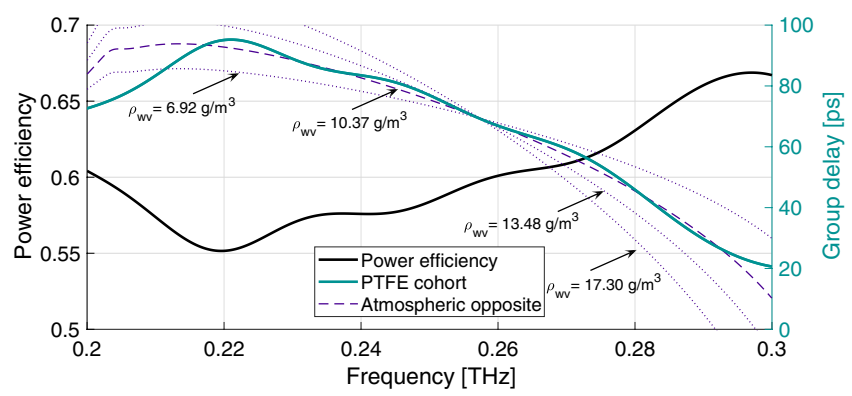

Figure 3. Analytic performance of PTFE-MGTI cohort. The dashed line indicates the target group delay, the opposite of atmospheric group delay at $\rho_{\mathrm{wv}}=10.37 \mathrm{~g} / \mathrm{m}^{3}$ for a $4 \mathrm{~km}$ propagation range. The dotted lines show how varying the atmospheric water vapor density for the $4 \mathrm{~km}$ link affects the target group delay. The increasing vapor densities given on the plot correspond to $40 \%, 60 \%, 80 \%$, and $100 \%$ relative humidity at $20{ }^{\circ} \mathrm{C}$ respectively. The thick black curve indicates the overall power efficiency. Plots show overall effects of four reflections from each MGTI of the cohort.

$90 \mu \mathrm{m}$ of air, reflector. In operation, the THz wave would reflect once from each MGTI to achieve full compensation. The cohort reflects more than $98 \%$ of incident power ( $0.03 \mathrm{~dB}$ insertion loss per MGTI per reflection) where conductor losses in the high-reflector constituted the majority loss mechanism. The cohort design also resulted in a good fit to the target group delay curve, as can be seen from Fig. 2.

As a second demonstration, a cohort of MGTIs based on polytetrafluoroethylene (PTFE) layers was also designed. The first MGTI of the cohort had a layering of $480 \mu \mathrm{m}$ of PTFE, $380 \mu \mathrm{m}$ of air, $510 \mu \mathrm{m}$ of PTFE, $250 \mu$ $\mathrm{m}$ of air, and finally the reflector. The second MGTI of the cohort had a layering of $430 \mu \mathrm{m}$ of PTFE, $750 \mu \mathrm{m}$ of air, $540 \mu \mathrm{m}$ of PTFE, $290 \mu \mathrm{m}$ of air, then the reflector. As before, the cohort was designed to compensate the dispersion produced by $4 \mathrm{~km}$ of propagation through the atmosphere with $\rho_{\mathrm{wv}}=10.37 \mathrm{~g} / \mathrm{m}^{3}(60 \%$ relative humidity at $20{ }^{\circ} \mathrm{C}$ ). Note that PTFE has a lower index than silicon (a constant index $n_{\text {PTFE }}=1.42+i 0.0032$ was assumed based on measurements), which reduces the reflections at the layer boundaries, resulting in less overall dispersion. This allowed us to more precisely match the atmosphere's dispersion profile for varying water vapor densities at the expense of cumulative dispersive power.

PTFE absorptive losses are not large in the THz range, but they accumulate when waves traverse the layers of the MGTIs multiple times, leading to greater overall insertion loss than a silicon-based device. Because of this, both material and reflector losses must be accounted for. Despite this added loss, the PTFE cohort is still highly effective at compensating atmospheric dispersion over shorter distances. This cohort of two MGTIs reflects more than $86 \%$ of incident power for a single pass through the cohort ( $0.3 \mathrm{~dB}$ insertion loss per MGTI per reflection). However, since its dispersive power is about $25 \%$ of the Si-based cohort, the THz beam must reflect from each PTFE-MGTI four times to compensate the target $4 \mathrm{~km}$ channel, which lowers the overall cohort power efficiency to $55 \%$ (minimum) or $2.6 \mathrm{~dB}$ insertion loss total, as shown in Fig. 3. An advantage of the PTFE-cohort, apparent from Fig. 3 , is that it exhibits a better fit to the target group delay curve.

To further demonstrate the MGTI cohorts, we calculated the time-domain waveforms for THz pulses before and after compensation. Again, we assumed plane-wave propagation of a THz pulse through the atmosphere at $\rho_{\mathrm{wv}}=10.37 \mathrm{~g} / \mathrm{m}^{3}$ over a distance of $4 \mathrm{~km}$. The initial transform-limited pulse was given a raised cosine spectrum centered at $0.25 \mathrm{THz}$, with a full-width-half-max (FWHM) bandwidth of $0.05 \mathrm{THz}$. After propagating through the atmosphere, this pulse was dispersed to $175 \%$ of its original width by GVD and reduced in amplitude by 9.1 times due to water vapor absorption losses, as shown by the electric-field waveforms in Fig. 4 . For determination of the pulse width, we assumed an approximately Gaussian profile of the pulse both before and after dispersion.

This dispersed pulse was compensated by both the Si- and PTFE-based MGTI cohorts. As before, compensation was achieved by either one (Si), or four (PTFE), normal-incidence reflections off the cohort. These atmospheric conditions represent a natural transition point between the silicon and PTFE approaches. The silicon cohort cannot be used for less compensation because there can be no less than one reflection, while the PTFE cohort is less desirable for more compensation because of accumulating losses. The analytic transfer functions of the cohorts were calculated and multiplied by the dispersed pulse spectrum in the frequency domain, then an inverse Fourier transform was used to obtain the time-domain output waveforms.

As shown in Fig. 4, the pulse went from $175 \%$ to $105 \%$ (for both Si and PTFE) of its original width. This significant reduction in pulse width corresponds to a $66 \%$ increase in spectral efficiency, $\eta_{\text {eff }}=B / \Delta f_{\text {ch }}{ }^{16}$, where $\Delta f_{\text {ch }}$ is the pulse bandwidth, and $B$ is the bit rate, which is inversely proportional to the pulse width. These numbers do not fully describe the effectiveness of the approach. Even a perfect dispersion compensation scheme cannot fully compensate for atmospheric dispersion, because some signal bandwidth is irreversibly lost due to absorption ${ }^{26}$. When this is accounted for, the Si-MGTI and PTFE-MGTI cohorts both achieve $>99 \%$ of the theoretically possible dispersion compensation, but the PTFE cohort is better, nearly reaching perfect compensation $(99.8 \%$ by calculation).

It is worth noting that the missing $\sim 1 \%$ of possible compensation for the Si cohort is manifested in the presence of "auxiliary pulses" on the compensated waveforms of Fig. 4. These pulses contain very little power and are not large enough to significantly contribute to ISI. Their presence may be linked back to the frequency-dependent oscillations in the cohort's group delay around the target group delay (See Fig. 2). In other words, they result from 


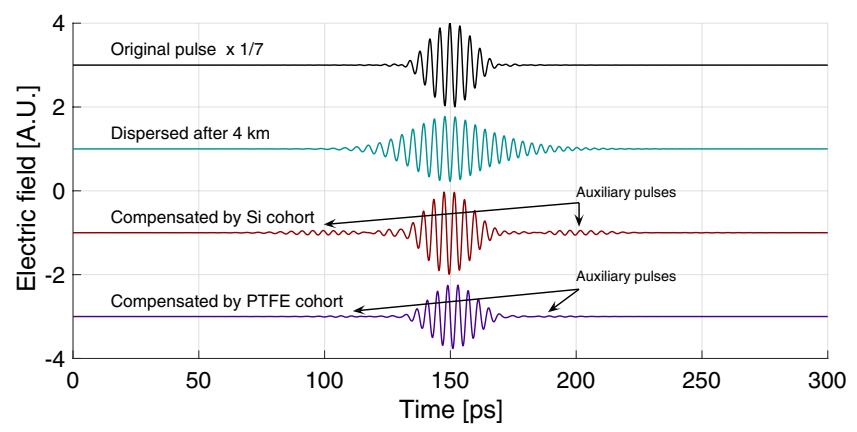

Figure 4. Time-domain terahertz pulses before and after propagation through atmosphere and compensators. The figure shows the original pulse (black), pulse after propagation through atmosphere (blue), pulse after SiMGTI cohort compensation (red), and pulse after PTFE-MGTI cohort (purple). All waveforms are shown to scale except the original pulse, which is scaled down in amplitude by 7 times to aid display. Auxiliary pulses are evident in compensated waveforms, as shown.

the non-zero derivative of the sum of group delays of the atmosphere and the MGTIs. The auxiliary pulses can be minimized by using a lower index material (such as PTFE) to construct the MGTIs. This reduces the lifetime of resonances within the MGTI layers, thus reducing the amplitude of the group delay oscillations. For example, both the group delay oscillations (Figs. 2 and 3) and the auxiliary pulses (Fig. 4) are much smaller in the PTFE cohort than in the Si cohort. Auxiliary pulses may also be suppressed by the addition of more layers to the MGTIs, or by the addition of more MGTIs to the cohort. However, given the exceptionally good match achieved with a small number of MGTIs and dielectric layers, these adjustments appear unnecessary. For longer range systems, such strategies might be employed with Si-based MGTIs to obtain every advantage in both optimized dispersion compensation and low loss.

It is also worth noting that the dispersion profile of the atmosphere is a function of atmospheric water vapor density (as shown in Fig. 3), thus the effectiveness of the MGTI cohort will be somewhat lessened whenever the vapor density deviates significantly from the design value. Repeating the time-domain calculations previously described for the PTFE cohort at the vapor densities shown in Fig. 3 yields the following results: at $\rho_{\mathrm{wv}}=6.92 \mathrm{~g} /$ $\mathrm{m}^{3}$, the input pulse is dispersed to $138 \%$ its original width, and compensated to $110 \%$ ( $79 \%$ of ideal). At $\rho_{\mathrm{wv}}=13.84 \mathrm{~g} / \mathrm{m}^{3}$, the input is dispersed to $205 \%$ its original width, and compensated to $114 \%$ (93\% of ideal). Finally, at $\rho_{\mathrm{wv}}=17.3 \mathrm{~g} / \mathrm{m}^{3}$, the input is dispersed to $232 \%$ its original width, and compensated to $138 \%(78 \%$ of ideal). These values are for a static cohort, however, it is possible to dynamically match the changing dispersion profile of the atmosphere by altering the arrangement of the MGTIs in the cohort, improving performance. This idea is explored in greater detail in the discussion.

\section{Experimental validation}

The analytic calculations were supported by both full wave simulations and experimental measurements, the latter detailed here. It was not possible to experimentally implement a $4 \mathrm{~km}$ channel with uniform and tuned atmospheric properties. But the absorptive and dispersive behaviors of the atmosphere are already well-understood in great detail and the models have been verified by experimentation ${ }^{12,27}$. Therefore, to validate the concept, it is sufficient to demonstrate only that an MGTI-based compensator can produce the target "atmospheric opposite" group delay in the bandwidth of interest. We elected to fabricate and measure one MGTI of the PTFE-cohort described above and measure it with reflection-mode terahertz time-domain spectroscopy (THz-TDS). This MGTI has an ideal structure of $480 \mu \mathrm{m}$ of PTFE, $380 \mu \mathrm{m}$ of air, $510 \mu \mathrm{m}$ of PTFE, $250 \mu \mathrm{m}$ of air, and finally an aluminum mirror. Fig. 5(b) shows the actual fabricated device alongside a schematic of its inner structure in Fig. 5(c). The MGTI diameter was made large enough $(\sim 12 \mathrm{~mm})$ to avoid clipping the THz beam focused onto its surface. Further fabrication and measurement details are given in the methods section below.

The measured and predicted time-domain traces are shown in Fig. 6 . This match is presented in the time-domain to better illustrate that the waveforms are nearly identical. This proves that the MGTI is behaving almost exactly like the model over the entire measured frequency range $(0.1-2.5 \mathrm{THz})$, both in phase and amplitude. A notable discrepancy between the measured and predicted waveforms is found at the earliest (leftmost) feature on the plot. This feature is the reflection off the first PTFE layer of the MGTI, and its slight time misalignment suggests that this layer was fabricated thinner than intended.

The measured data can be analyzed in the frequency domain to extract the group delay and compare to model predictions, as shown in Fig. 7. Again, there is good agreement between the modeled and measured values, which confirms both our analytical calculations and the validity of the GVD compensation approach. We note that these curves represent the effect of only one reflection from one MGTI of the cohort, hence the graphs of Figs. 3 and 7 are different. Discrepancies between measured and predicted results arise primarily because our fabrication techniques could not exactly produce the desired layer thicknesses nor uniformity. Specifically, the first PTFE layer appears to have been fabricated as $450 \mu \mathrm{m}$, or $30 \mu \mathrm{m}$ thinner than intended. When the model is modified to account for this error, the predicted and measured group delay profiles are in excellent agreement, as shown by the trace labeled "Modeled-ERR" in Fig. 7. The experimental results confirm that our analytic calculations are accurate, and that such devices are readily designed and fabricated. 


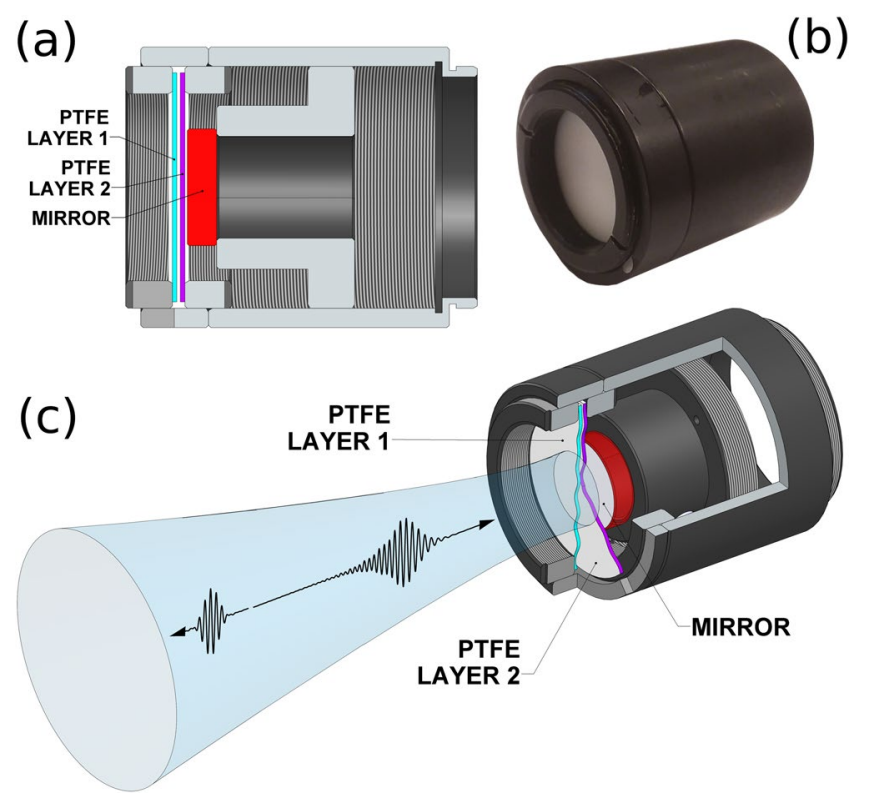

Figure 5. A prototype terahertz dispersion compensating MGTI. (a) cross-section drawing of designed and fabricated MGTI, (b) fabricated MGTI, with PTFE first layer visible (c) scale cutaway drawing of fabricated MGTI showing internal layering. Blue tube depicts focused input/output terahertz beam.

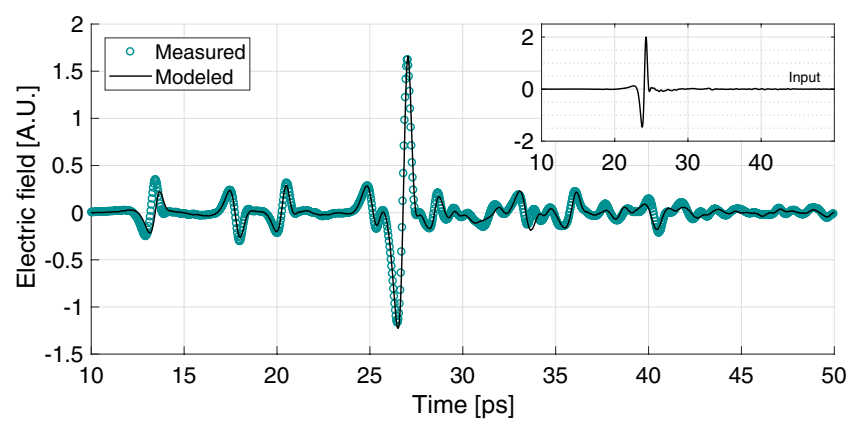

Figure 6. Measured and predicted time-domain waveforms for THz pulses reflected from the PTFE MGTI shown in Fig. 5. The inset shows the measured reference pulse, obtained by removing the two PTFE layers in front of the reflector.

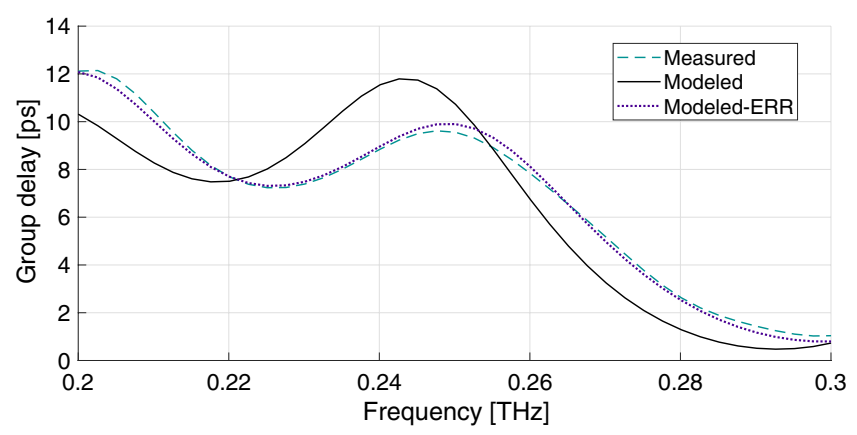

Figure 7. Measured and predicted group delay for THz waves reflected from the PTFE MGTI shown in Fig. 5.

\section{Discussion}

Since the THz wireless channel would generally be dynamic, both in terms of atmospheric properties (weather) and signal range, it is important to address the adaptability of our approach to changing channel conditions. The dispersion of an MGTI cohort can be dynamically adapted most easily by altering the number of times the $\mathrm{THz}$ 


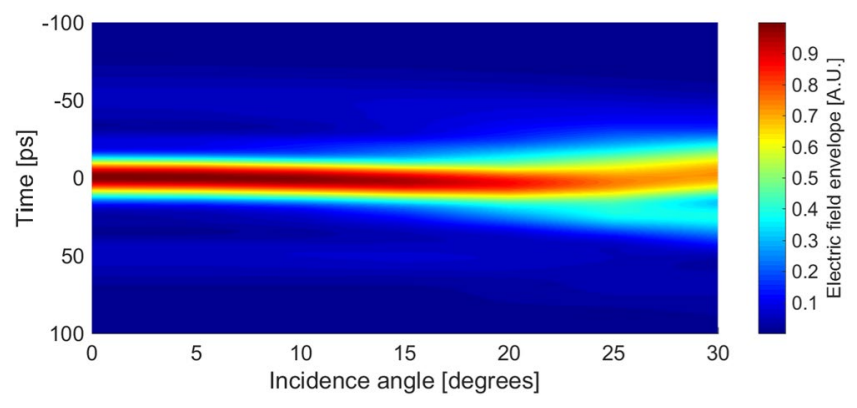

Figure 8. Time-domain compensated THz pulse evolution as a function of incidence angle on the Si-MGTI cohort, assuming p-polarization. Unlike Fig. 4, the normalized envelope of the electric field is shown for clarity. The pulse begins to noticeably disperse in time when the obliquity angle exceeds $15^{\circ}$.

beam is reflected from it. By mechanically adjusting the angle and position of the individual cohort members, the dispersed signal can be made to undergo more or fewer reflections from each MGTI as desired, which corresponds to a discrete increase or decrease in the level of dispersion achieved. By using a low-index dielectric MGTI in the cohort, the resolution of these discrete changes can be very fine, making it possible to compensate effectively a continuous range of changing atmospheric conditions and ranges. Even though changing the number of reflections would be a slow tuning procedure, it is more than sufficient for this application because channel conditions also change quite slowly.

Altering the physical geometry of the signal path to either add or reduce reflections will also mean changing the angle at which the wave is incident on each MGTI. However, the complex reflection coefficient of a stratified dielectric is a function of incidence angle and polarization ${ }^{23}$. Thus, there is a limit to how obliquely the signal may strike each MGTI before the cohort will no longer adequately compensate the atmospheric dispersion. Our calculations show that the silicon cohort described above will still achieve $90 \%$ or greater of the possible dispersion compensation as long as the incidence angle for all MGTIs is less than $15^{\circ}$ off normal in either direction, for both $s$ and $p$ polarizations. This is sufficiently flexible for the mechanical tuning techniques described above, and it also illustrates the excellent robustness of the technique to practical alignment errors. The dependence of compensated pulse shape on incidence angle for the Si cohort is shown in Fig. 8 for a p-polarized (worst case) incident pulse.

An important attribute of this device is that its effectiveness does not depend on the form or modulation of the incident $\mathrm{THz}$ signal. Though the figures and analyses show the dispersion and subsequent compensation of transform-limited pulses (as would be used in impulse radio), continuous-wave modulation schemes such as quadrature amplitude modulation and frequency-shift keying will suffer dispersion in like manner, and will be compensated with the same effectiveness. This is because GVD is a function of channel properties only. Regardless of modulation, any $\mathrm{THz}$ signal with a given bandwidth, having suffered dispersion in the atmosphere, will be compensated by interacting with a device exhibiting a group velocity dispersion opposite the atmosphere over that same bandwidth.

In conclusion, we have presented an effective method for compensating atmospheric dispersion in long-distance $\mathrm{THz}$ wireless links to thereby maximize data transmission rates to their fundamental limit. To our knowledge, this device is the first of its kind, and this work represents an important step toward the implementation of point-to-point and point-to-mobile THz wireless links. Furthermore, compensating devices such as the ones presented here are highly effective in terms of both complete dispersion management and low loss, are inexpensive to fabricate, and are surprisingly tolerant to manufacturing errors. Though not discussed in this paper, they may also be easily optimized for oblique incidence, which allows for flexibility in how such devices are physically incorporated into $\mathrm{THz}$ communication systems.

\section{Methods}

Atmospheric modeling. The atmosphere was modeled using molecular response theory (MRT), in conjunction with molecular resonance data from the HITRAN database. For each $\mathrm{H}_{2} \mathrm{O}$ molecular resonance in the database, the frequency, resonance strength, and broadening factor was extracted. Resonances too weak or too far distant to influence the frequency range of interest were ignored. MRT was used to model the broadened lineshapes of the remaining resonances. MRT, rather than van-Vleck-Weisskopf or Full Lorentzian models, is used to model the broadened resonance lineshapes because MRT produces a superior match to experimental data ${ }^{11,12,27}$. The exact shape of the broadened resonance lines produced by the MRT model depends heavily on the temperature, pressure, and water vapor density of the atmosphere, so these parameters must be specified before modeling. After the broadened lineshapes are determined, they are summed up over the frequency range of interest to produce the frequency-dependent transfer function of the atmosphere. Finally, multiplying the transfer function of the atmosphere by the Fourier transform of the input waveform (which is assumed to be a plane wave) yields the frequency-domain representation of the dispersed waveform. Converting this back to the time domain gives the dispersed pulse.

Experimental setup. Our reflection-mode THz-TDS setup permits phase-coherent measurements of the sample and reference at normal incidence and is illustrated in Fig. 9. It is modified from the standard transmission 


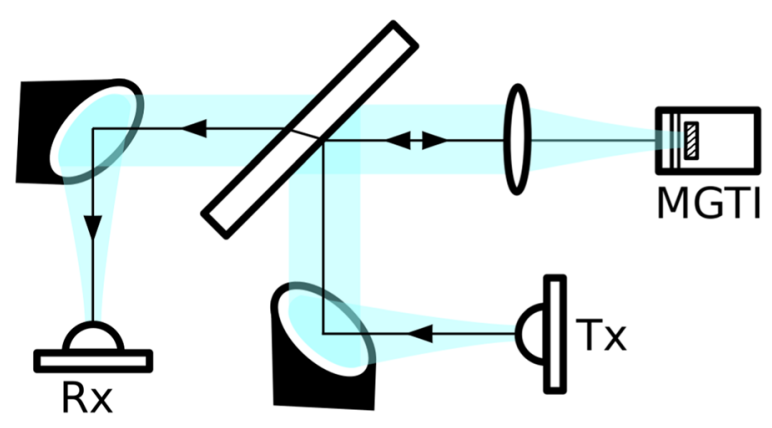

Figure 9. Reflection-mode THz-TDS setup for normal-incidence, phase-coherent measurements. The system is confocal and produces a frequency-independent beam waist with a planar phase front at the MGTI sample. The blue shaded areas illustrate the terahertz beam profile (not to scale). Labels "Tx" and "Rx" refer to the terahertz transmitter and receiver.

setup described in reference ${ }^{28}$. Generated terahertz pulses are collimated by an off-axis paraboloidal mirror to a 3 $\mathrm{mm}$ thick high-resistivity float-zone silicon beamsplitter. From here, they are reflected toward and focused onto the sample at normal incidence using a polyethylene lens. At the sample, the frequency-independent beam waist diameter is $\sim 7 \mathrm{~mm}$. After reflecting off the sample, the broadband beam is again collimated by the polyethylene lens and then passes through the beamsplitter. It is finally focused into the THz receiver by another off-axis paraboloidal mirror. The entire system is confocal to maximize power transfer and ensure a frequency-independent beam waist at the sample.

The thickness of the silicon beamsplitter is chosen to be large so that the initial pulse and subsequent features in the time-domain are easily separable from later echo pulses, which are caused by multiple reflections within the beamsplitter. If the beamsplitter is too thin, the time-domain features introduced by the sample will overlap with the features introduced by the beamsplitter, significantly increasing the difficulty of extracting the sample parameters. Obtaining the transfer function of the MGTI requires knowledge of the spectrum of the incident wave. The incident wave is measured by removing the two PTFE layers in front and measuring only the aluminum mirror of the MGTI sample. The MGTI is mounted such that the mirror does not move when the PTFE layers are added between sample and reference measurements, thus establishing a fixed phase reference for proper determination of the transfer function.

Fabrication of the sample. The sample was constructed from $20 \mathrm{~mm}$ diameter PTFE sheets, manually spaced using the apparatus shown in Fig. 5. PTFE sheets of the desired thicknesses were not commercially available, and thus had to be fabricated by hand. Consequently, though the fabricated layer thicknesses and spacings were close to the intended values, the thickness of the layers and spacings were not ideal. All layers were within the $\pm 5 \mu \mathrm{m}$ tolerance, with the exception of the first PTFE layer, which measurements indicated was about $30 \mu \mathrm{m}$ thinner than intended. According to our numerical analyses, these fabrication errors - with the exception of the first layer - are small enough to not significantly affect the group delay profile of the sample.

\section{Data availability}

The datasets generated and analysed during the current study are available from the corresponding author on reasonable request.

Received: 29 July 2019; Accepted: 13 March 2020;

Published online: 02 April 2020

\section{References}

1. Wells, J. Faster than fiber: The future of multi-G/s wireless. IEEE Microwave Magazine 10, 104-112, https://doi.org/10.1109/ mmm.2009.932081 (2009).

2. Cherry, S. Edholm's law of bandwidth. IEEE Spectrum 41, 58-60, https://doi.org/10.1109/mspec.2004.1309810 (2004).

3. Song, H.-J. \& Nagatsuma, T. Present and future of terahertz communications. IEEE Transactions on Terahertz Science and Technology 1, 256-263, https://doi.org/10.1109/tthz.2011.2159552 (2011).

4. Ma, J., Shrestha, R., Moeller, L. \& Mittleman, D. M. Invited article: Channel performance for indoor and outdoor terahertz wireless links. APL Photonics 3, 051601, https://doi.org/10.1063/1.5014037 (2018).

5. Global mobile data traffic forecast update, 2017-2022. https://www.cisco.com/c/en/us/solutions/collateral/service-provider/visualnetworking-index-vni/white-paper-c11-738429.html (2019). Accessed: 2019-7-22.

6. Dastjerdi, A. V. \& Buyya, R. Fog computing: Helping the internet of things realize its potential. Computer 49, 112-116, https://doi. org/10.1109/mc.2016.245 (2016).

7. Cortés, R., Bonnaire, X., Marin, O. \& Sens, P. Stream processing of healthcare sensor data: Studying user traces to identify challenges from a big data perspective. Procedia Computer Science 52, 1004-1009, https://doi.org/10.1016/j.procs.2015.05.093 (2015).

8. Mumtaz, S. et al. Terahertz communication for vehicular networks. IEEE Transactions on Vehicular Technology 66, 5617-5625, https://doi.org/10.1109/TVT.2017.2712878 (2017).

9. Chen, Z. et al. A survey on terahertz communications. China Communications 16, 1-35 (2019).

10. Yang, Y., Mandehgar, M. \& Grischkowsky, D. R. Broadband THz pulse transmission through the atmosphere. IEEE Transactions on Terahertz Science and Technology 1, 264-273, https://doi.org/10.1109/TTHZ.2011.2159554 (2011).

11. Harde, H., Cheville, R. A. \& Grischkowsky, D. Terahertz studies of collision-broadened rotational lines. The Journal of Physical Chemistry A 101, 3646-3660, https://doi.org/10.1021/jp962974c (1997). 
12. Yang, Y., Mandehgar, M. \& Grischkowsky, D. Determination of the water vapor continuum absorption by THz-TDS and Molecular Response Theory. Opt. Express 22, 4388-4403, https://doi.org/10.1364/OE.22.004388 (2014).

13. Xin, X., Altan, H., Saint, A., Matten, D. \& Alfano, R. R. Terahertz absorption spectrum of para and ortho water vapors at different humidities at room temperature. Journal of Applied Physics 100, 094905, https://doi.org/10.1063/1.2357412 (2006).

14. Hill, R. J. Dispersion by atmospheric water vapor at frequencies less than 1 THz. IEEE Transactions on Antennas and Propagation 36, 423-430, https://doi.org/10.1109/8.192126 (1988).

15. Mandehgar, M., Yang, Y. \& Grischkowsky, D. Experimental confirmation and physical understanding of ultra-high bit rate impulse radio in the THz digital communication channels of the atmosphere. Journal of Optics 16, 094004, https://doi.org/10.1088/20408978/16/9/094004 (2014).

16. Agrawal, G. P. Fiber-Optic Communication Systems (Wiley-Blackwell, 2010), 4 edn.

17. $\mathrm{Wu}$, Q. et al. A $21 \mathrm{~km} 5 \mathrm{Gbps}$ real time wireless communication system at $0.14 \mathrm{THz}$. In 2017 42nd International Conference on Infrared, Millimeter, and Terahertz Waves (IRMMW-THz), 1-2, https://doi.org/10.1109/IRMMW-THz.2017.8066870 (2017).

18. Hirata, A. et al. $5.8-\mathrm{km} \mathrm{10-Gbps} \mathrm{data} \mathrm{transmission} \mathrm{over} \mathrm{a} \mathrm{120-GHz-band} \mathrm{wireless} \mathrm{link.} \mathrm{In} 2010$ IEEE International Conference on Wireless Information Technology and Systems, 1-4, https://doi.org/10.1109/ICWITS.2010.5611945 (2010).

19. Wang, C., Changxing Lin, Qi Chen, Xianjin Deng \& Jian Zhang. $0.14 \mathrm{THz}$ high speed data communication over 1.5 kilometers. In 2012 37th International Conference on Infrared, Millimeter, and Terahertz Waves, 1-2, https://doi.org/10.1109/IRMMWTHz.2012.6380109 (2012).

20. Ducournau, G. et al. Ultrawide-bandwidth single-channel 0.4-THz wireless link combining broadband quasi-optic photomixer and coherent detection. IEEE Transactions on Terahertz Science and Technology 4, 328-337, https://doi.org/10.1109/TTHZ.2014.2309006 (2014).

21. Antes, J. et al. $220 \mathrm{GHz}$ wireless data transmission experiments up to $30 \mathrm{gbit} / \mathrm{s}$. In 2012 IEEE/MTT-S International Microwave Symposium Digest, 1-3, https://doi.org/10.1109/MWSYM.2012.6259561 (2012).

22. Ducournau, G. et al. THz communications using photonics and electronic devices: the race to data-rate. Journal of Infrared, Millimeter, and Terahertz Waves 36, 198-220, https://doi.org/10.1007/s10762-014-0112-x (2015).

23. Born, M. \& Wolf, E. Principles of Optics: Electromagnetic Theory of Propagation, Interference and Diffraction of Light (Cambridge Univ. Press, 2002), 7 edn.

24. Mandehgar, M., Yang, Y. \& Grischkowsky, D. Atmosphere characterization for simulation of the two optimal wireless terahertz digital communication links. Opt. Lett. 38, 3437-3440, https://doi.org/10.1364/OL.38.003437 (2013).

25. Gordon, I. et al. The HITRAN2016 molecular spectroscopic database. Journal of Quantitative Spectroscopy and Radiative Transfer 203, 3-69, https://doi.org/10.1016/j.jqsrt.2017.06.038 (2017).

26. Mandehgar, M. \& Grischkowsky, D. R. Understanding dispersion compensation of the THz communication channels in the atmosphere. IEEE Photonics Technology Letters 27, 2387-2390, https://doi.org/10.1109/LPT.2015.2466559 (2015).

27. O'Hara, J. F. \& Grischkowsky, D. R. Comment on the veracity of the ITU-R recommendation for atmospheric attenuation at terahertz frequencies. IEEE Transactions on Terahertz Science and Technology 8, 372-375, https://doi.org/10.1109/ TTHZ.2018.2814343 (2018)

28. van Exter, M. \& Grischkowsky, D. R. Characterization of an optoelectronic terahertz beam system. IEEE Transactions on Microwave Theory and Techniques 38, 1684-1691, https://doi.org/10.1109/22.60016 (1990).

\section{Acknowledgements}

The authors gratefully acknowledge Prof. Daniel Grischkowsky for his helpful perspectives and suggestions on this manuscript.

\section{Author contributions}

J.O. and K.S. conceived and/or performed the dispersion compensation approach, the theoretical analysis and simulations, the sample design methods, and the measurement method. K.S. fabricated the sample and conducted the measurements. K.S., J.O., and S.E. analysed the data and wrote the manuscript.

\section{Competing interests}

Dr. John F. O'Hara and Karl L. Strecker are named as inventors on provisional patent application number US $62 / 898,358$, which claims protection for THz GVD manipulation by means of stratified media as described within this manuscript, and which is held by Oklahoma State University. Dr. Sabit Ekin declares no competing interests.

\section{Additional information}

Correspondence and requests for materials should be addressed to J.F.O.

Reprints and permissions information is available at www.nature.com/reprints.

Publisher's note Springer Nature remains neutral with regard to jurisdictional claims in published maps and institutional affiliations.

Open Access This article is licensed under a Creative Commons Attribution 4.0 International License, which permits use, sharing, adaptation, distribution and reproduction in any medium or format, as long as you give appropriate credit to the original author(s) and the source, provide a link to the Creative Commons license, and indicate if changes were made. The images or other third party material in this article are included in the article's Creative Commons license, unless indicated otherwise in a credit line to the material. If material is not included in the article's Creative Commons license and your intended use is not permitted by statutory regulation or exceeds the permitted use, you will need to obtain permission directly from the copyright holder. To view a copy of this license, visit http://creativecommons.org/licenses/by/4.0/.

(c) The Author(s) 2020 